\title{
Antonela Marić \\ Il giallo con il gusto del gioco
}

\section{Le lunghe ombre della morte di Veit Heinichen}

Riassunto: In questo contributo si riflette inizialmente sulle trasformazioni e le varianti che contraddistinguono il giallo e i suoi protagonisti, sia quelli dalla parte della legge sia i loro antagonisti. Entrambi si caratterizzano sempre più di frequente per tratti positivi e negativi che li rendono testimoni di un processo di emancipazione rispetto alle formule classiche del genere. L'indagine prosegue focalizzandosi su Trieste, città di 'frontiera' per antonomasia, con la sua complessa realtà, ed evidenzia come essa possa essere indagata e rappresentata anche attraverso il genere noir; in particolare quella sua variante 'mediterranea' cui appartengono i romanzi di Veit Heinichen, tedesco di nascita e triestino d'adozione, che offrono il ritratto variegato di una Trieste realistica, riconoscibile, ma nel contempo gravida di segreti relativi ad un passato che non cessa di condizionare il presente. A dimostrazione di come, rispetto alla classica triade tipica del genere, in questo caso vi sia da aggiungere un quarto ed essenziale elemento rappresentativo, lo spazio.

\section{The crime novel and its taste for play: Veit Heinichen's Le lunghe ombre della morte}

\begin{abstract}
This essay first discusses the transformations and variants of the crime novel and its protagonists, be it the characters representing the law or their antagonists. Both are increasingly characterized by positive and negative traits which make them witnesses of a process of emancipation from classical formulas of the genre. The analysis then goes on to focus on Trieste, 'border city' par excellence, and its complex realities, illustrating how these can also be represented in the crime novel genre, particularly in its 'Mediterranean' variant. The crime novels by German-born and Trieste-based Veit Heinichen belong to this specific subgenre and draw a complex, realistic picture of Trieste, which remains recognisable while revealing, at the same time, numerous secrets stemming from a past that never ceases to condition the present. The author argues that in this specific case, the genre's classical triad must be extended to include a fourth essential element: space.
\end{abstract}

ว Open Access. ( 2020 Antonela Marić, published by De Gruyter. (c) BY-NC-ND This work is licensed under the Creative Commons Attribution-NonCommercial-NoDerivatives 4.0 License.

https://doi.org/10.1515/9783110640069-018 


\section{Introduzione}

Leonardo Sciascia, in uno dei suoi numerosi scritti sul giallo, ${ }^{1}$ evidenzia (non senza ironia) l'esasperata frequenza del genere giallo con il costante lievitare delle pubblicazioni e dei volumi sul poliziesco, noir, thriller e simili. L'autore, lamentandosi delle tendenze attuali del giallo, opera una netta distinzione tra il «giallo con il gusto del gioco» (annoverandovi i gialli classici di tipo holmesiano e simili in grado di offrire divertimento ed evasione e di proporre al lettore la ricostruzione e nel contempo anche la risoluzione di un labirinto mentale «algebrico, parodiato e complicato») e quel giallo ${ }^{2}$ leggero e superficiale che si perde nella morbosità dei suoi effetti, nel sadismo delle sue rappresentazioni e nell'eccesso pornografico delle sue scene.

È ben noto che il giallo con i suoi sottogeneri non solo si è infiltrato in tempi recenti nelle letterature europee, perlopiù in quella tedesca, francese e italiana, ma è diventato forse il genere più suscettibile di trasformazioni e slittamenti sui generis. Il lungo percorso partito con il primo detective poeiano che ha avuto inizio durante il romanticismo americano ora sembra più incisivo e gagliardo che mai. Oggi viene particolarmente valorizzato il percorso creativo di autori come Georges Simenon o Agatha Christie, che hanno contribuito alla popolarità del genere grazie a personaggi canonici come Poirot, Marple o Maigret. Tuttavia non sarebbe giusto non riconoscere l'enorme influsso che il giallo americano con i suoi delitti, poliziotti e investigatori privati ha esercitato sul pubblico. Alla diffusione del genere hanno indubbiamente contribuito le serie televisive che hanno raggiunto un'enorme popolarità a livello globale come La signora in giallo, con la scrittrice-detective Jessica Fletcher (forse una delle serie più lunghe, andata in onda per ben dodici stagioni) o Il tenente Colombo, degli anni Sessanta sospesa nel 2003; Miami Vice (1984-1990) con Don Johnson che ha rivoluzionato il genere poliziesco; Magnum (1980-1988) con Tom Selleck, o Crime Story (19861988) con l'indimenticabile Dennis Farina nel ruolo di Mike Torello; The Sopranos (1999-2007) con James Gandolfini nella parte di Tony Soprano, boss della mafia italoamericana, per ricordarne solo alcuni.

Il giallo cosidetto classico o ad enigma, definito a volte come giallo tradizionale, ha subito un'evoluzione importante, uscendo dallo spazio chiuso e

1 Cfr. Leonardo Sciascia, Il metodo di Maigret e altri scritti sul giallo, Milano, Adelphi, 2018.

2 Dice al riguardo Sciascia: «L'autore non si concentra pertanto nel giuoco ingegnoso tutto teso allo scoglimento sorprendente: e il racconto resta poliziesco soltanto per il fatto che la polizia c'entra in qualche modo, magari nascondendo il delinquente nelle sue file, e non perché alla polizia è demandato lo scoglimento, che vorremmo dire edificante, dell'intrigo» (ivi, p. 16). 
limitato (della camera dell'albergo o della villa privata) all'aperto, modernizzando metodologie e argomenti. Lo scrittore diviene consapevole della necessità di introdurre i cosidetti «schiaffi emotivi», ${ }^{3}$ per dirla con Highsmith, i cosiddetti ribaltamenti plurimi, nella costruzione della suspence. Con lo sviluppo della tecnologia, si creano nuovi metodi, si accelera il ritmo narrativo, si sviluppano nuove tecniche investigative, rendendo gli indizi minuziosamente raccolti dalle simpatiche zitelle-scrittrici-detective o interpretati dai poliziotti dall'aspetto shabby e un po' obsoleti, indipendentemente da quanto amabili fossero questi protagonisti. L'immaginazione del lettore si è arrichita delle nuove conoscenze e delle nuove aspettative che potevano essere soddisfatte solo uscendo dall'ambiente sigillato della camera d'albergo. Cambia anche il gioco di ambivalenze di cui il delinquente e il poliziotto fanno parte, perché in un secondo momento è come se i due si scambiassero i ruoli, e la formula si rovescia a tal punto che il poliziotto diventa quasi un delinquente, mentre il delinquente cambia il modo d'essere. Patricia Highsmith addirittura attribuisce ai suoi personaggi psicopatici e assassini anche qualità positive come la generosità o l'interesse per l'arte, «qualità che possono anche rappresentare un divertente contrasto con i suoi tratti criminali e omicidi». ${ }^{4}$

Banalizzando, si potrebbe confermare la loro emancipazione, in quanto diventano decisivi nell'identificazione del nuovo genere. A titolo di esempio menzioneremo qui soltanto Maurizio De Giovanni e I bastardi di Pizzofalcone con Alessandro Gassmann nei panni del commissario Giuseppe Lojacono, a nostro avviso emblematico perché rappresenta forse al meglio i personaggi «delinquenti dalla parte della legge» a cui accenna Sciascia nei suoi appunti sul genere giallo. ${ }^{5}$ La squadra di Pizzofalcone è composta da un gruppo di poliziotti violenti, con gravi problemi familiari, conosciuti per l'esagerato uso di armi. Il gruppo dei 'vinti' (si potrebbe tranquillamente usare il termine verghiano) vive una serie di tensioni anche nella vita professionale, si trova in costante attrito con il mondo circostante, e avverte anche forti conflitti interiori e personali, per cui questi protagonisti non hanno più niente da perdere e quindi agiscono senza remore né

3 Riflettendo sulle tipologie di suspence Patricia Highsmith sottolinea la rilevanza dell'esperienza emotiva nel lettore (Id., Come si scrive un giallo. Teoria e pratica della suspence, Roma, Edizioni minimum fax, 2015, p. 34).

4 Patricia Highsmith, Come si scrive un giallo. Teoria e pratica della suspence, cit., p. 55.

5 «Tutta l'emozione è nell'ambivalenza sentimentale che il giuoco tiene sospeso, drammaticamente, fino all'epilogo: che è sempre, nell'impressione del lettore, fiacco e delusivo - e da ciò, a lettura finita, il vuoto dell'insoddisfazione, e il bisogno delle nuove letture nello stesso senso, per rinnovare la stessa emozione» (L. Sciascia, Il metodo di Maigret e altri scritti sul giallo, cit., pp. 24-25). 
limitazioni. Questi nuovi eroi, rimasti incompiuti nella vita privata, sono immuni da ogni compromesso, perché guidati dalla rabbia e rispettosi solo delle regole della squadra. Ricordando i protagonisti cosidetti hard-boiled, come Mike Hammer di Mickey Spillane, non si potrebbe dire che i 'bastardi' per il loro modo di agire violento siano personaggi senza precedenti. Tuttavia è il loro modo di essere tutt'uno con la loro squadra, ormai diventata la loro famiglia, l'agire disinteressato e nonconformista nei confronti della violenza e della corruzione, l'inosservanza delle regole, il rifiuto delle consuetudini moralistiche, che li rende unici e rappresentativi del noir. E mentre gli antieroi come Dirty Harry o Mike Hammer hanno conservato l'immagine del poliziotto duro, con «la rivoltella sotto l'ascella e pronti a tirarla fuori in un lampo; nervosi, scazzottatori, un tantino loschi e perversi», ${ }^{6}$ l'immagine di questi ragazzi fondamentalmente solitari, i legami che uniscono e accomunano il gruppo dei 'bastardi' (ancora una volta qui citati a titolo di esempio), sono molto più forti ed omertosi, quasi di tipo camorristico.

Dobbiamo concordare ancora una volta con Sciascia e riconoscere l'abbandono della formula tradizionale ${ }^{7}$ del giallo che consisterebbe in tre elementi cardine: il delitto, il delinquente e il detective. Il nuovo giallo, quello noir, ${ }^{8}$ se si vuole il noir mediterraneo, è caratterizzato perlopiù dalla natura diabolica e sostanzialmente nera dei suoi temi e dei suoi eroi-antieroi, ponendosi l'obiettivo di raccontare e addirittura di smascherare la realtà carica di problemi sociali, di scoprire ogni singola verità e di denunciare i rapporti di corruzione nel contesto politico e sociale attuale, inserendoli nel mondo dell'alta finanza e delle organizzazioni clandestine. L'investigatore moderno ormai si muove e agisce ai margini della legge, spesso infrangendola senza troppa esitazione. D’altro lato, l'autore noir intende fotografare la realtà ponendo l'eroe-antieroe nel contesto urbano in cui si muove e compie le sue attività più facilmente, nell'ambiente che conosce bene, magari nell'area geografica di provenienza, il che comporta l'inserzione di specificità geografiche e storiche, costumi e abitudini. Si pensi alla Sicilia del commissario Montalbano di Andrea Camilleri, alla Napoli di Maurizio De Giovanni, alla Milano di Dario Crapanzano, alla Roma di Carlo Emilio Gadda, alla Bologna di Carlo Lucarelli, e a tante altre città che fungono da sfondo e da campo di battaglia contro il malaffare organizzato. Infine, tornando al Nord

\footnotetext{
6 L. Sciascia, Il metodo di Maigret e altri scritti sul giallo, cit., p. 68.

7 Ivi, pp. 28-29.

8 Cfr. Massimo Carlotto, The Black Album. Il noir tra cronaca e romanzo. Conversazione con Marco Amici, Roma, Carocci, 2012.
} 
troviamo lo sfondo perfetto, quello triestino, offerto dalla splendida e strabiliante serie di romanzi noir di Veit Heinichen.

\section{Mein Raum ist Europa!}

Trieste è una città 'di frontiera', città crocevia del continente europeo, che ha saputo accogliere varie etnie e culture, amalgama di tradizione e di modernità, ed è stata al centro di drammatiche vicende storiche. Trieste, vista dagli occhi di Veit Heinichen, è indubbiamente uno spazio tipicamente europeo; è il mondo in cui l'autore vive e che vuole raccontare, e che ha bisogno di conoscere e di capire. Lo afferma lui stesso in un'intervista del $2009,{ }^{9}$ dichiarando che da autore si ispira a vari luoghi della città, inserendo i suoi protagonisti in ristoranti e in bar che conosce bene e che visita spesso, in un tentativo di raccontare la realtà e di trasmettere al lettore il mondo reale che condivide con i suoi protagonisti. Il giallo nella forma e nella struttura è per questo motivo la soluzione ragionevole, poiché, secondo Heinichen, il genere giallo è da considerarsi per molti aspetti uno specchio della realtà, di un'epoca e di uno spazio. I protagonisti del noir, di varia estrazione sociale, appaiono assolutamente reali. Alessandro Perissinotto, ${ }^{10}$ parlando del successo massificato del giallo, accenna alla crisi di credibilità e al profondo senso di insicurezza, addirittura al caos. E mentre l'autore si interessa soprattutto alla dimensione psicologica del protagonista, cercando di avvicinarlo al lettore, questi si identifica con il protagonista e si pone anche dalla parte degli esclusi, entro una realtà colma di deviazioni.

Il nostro autore, inoltre, intende focalizzarsi sulle conseguenze del crimine, indagare gli spazi in cui questo viene compiuto, le sue manifestazioni, cercando di esaminarne le implicazioni sociali e la subcultura di cui è il prodotto. Questo è lo sfondo dei romanzi di Veit Heinichen che, da triestino adottivo di origini germaniche, conosce bene la storia socio-politica del Raum carsico, segnato profondamente dalle drammatiche vicende storiche dell'ultimo secolo. In questo senso l'autore si rende conto delle radicali trasformazioni subite da Trieste negli

9 (consultato il 10 ottobre 2019).

10 «E poiché ogni desiderio nasce da una mancanza, bisogna ammettere che il successo massificato verso questo genere letterario nasce da una crisi di credibilità e da un profondo senso di insicurezza, e il giallo con il suo finale consolatorio riporta l'ordine lì dove il delitto ha creato il caos. Ciò che ci manca nella realtà e che ricerchiamo nella fiction poliziesca non è soltanto una società dove il crimine venga punito, ma è un mondo di verità» (Alessandro Perissinotto, La società dell'indagine. Riflessioni sopra il successo del poliziesco, Milano, Bompiani, 2008, p. 4). 
ultimi secoli che, come traspare dai suoi romanzi, non riguarderebbero solo la città e la sua periferia ma che avrebbero avuto ripercussioni in tutto il sud-est europeo. Il noir per Heinichen si trasforma in uno strumento utile per indagare i residui e le anomalie del passato, e cercare di capirne i nessi con il presente.

In Le lunghe ombre della morte troviamo l'ambiente triestino, definito dall'autore la «rosa dei venti», ${ }^{11}$ con dinamiche che, nel lasso di tempo intercorso dal secondo dopoguerra ad oggi, non appaiono mutate. La città con i suoi segreti rimane «l'ombelico del mondo», molto orientata verso sé stessa. La mentalità e le abitudini non sembrano molto diverse. Il presente è carico di segreti che pesano sull'attualità, come se, per un gioco cosmico, il tempo e con esso la città, si fossero bloccati in una sorta di vacuum temporale.

Il protagonista del libro è Proteo Laurenti, commissario di polizia, che osserva la città con cinico realismo. Le sue indagini lo portano a incappare in due casi rimasti irrisolti negli anni Settanta: la morte di un collezionista, bruciato vivo in un magazzino di armi e sepolto in fretta senza l'autopsia, e l'omicidio di un professore omosessuale. Ambedue i casi vedono coinvolta una giovane australiana, figlia di emigrati triestini, che si reca a Trieste per sistemare le pratiche dell'eredità dopo la morte della zia. La situazione a Trieste nel 1954, quando i genitori vi sono emigrati, era difficile. Tuttavia, per la giovane protagonista, Trieste continua ad essere un ricordo avvolto nel mito, coltivato sin dall'infanzia con nostalgia. Heinichen costruisce una città di contrasti, la cui periferia con «piccole case in pietra, addossate le une alle altre per via della bora», un "piccolo torrente fiancheggiato da acacie, pioppi, salici e aceri», un «sentiero sassoso che conduce nella valle», sembra tranquilla e invitante. Le viene opposto l'ambiente molto meno idilliaco e molto più impersonale del centro, gli imponenti palazzi dell'epoca neoclassica che irradiano un calore enorme, come «accumulatori di energia solare». Ė piena estate, così che per le strade regna la calma, ma «negli sportelli dentro i palazzi le attività fervevano» ${ }^{12}$ e si lavora intensamente anche nella zona industriale, dove c'è la ferriera, il porto del carbone, con le gigantesche gru da carico. L'insensatezza e la crudeltà del consumismo sono presenti anche nei mestieri tradizionali, che dovrebbero escludere ogni traccia di corruzione o di violenza:

Veterinari si chiamavano quelli che praticavano iniezioni dopanti al bestiame incapace ormai di stare in piedi, tollerando impassibili che gli animali sfiniti venissero incatenati per una zampa o per le corna e issati a bordo dalla gru se non ce la facevano a salire da soli. Che i bovini muggissero per il dolore o la sete, a chi lavorava in quel posto sembrava una cosa

11 Veit Heinichen, Le lunghe ombre della morte, Roma, Edizioni e/o, 2006.

12 Ivi, p. 88. 
normale, proprio come a chi ne traeva guadagno grazie al premio corrisposto dall'Unione europea per ogni capo che non lasciava morto l'Europa. ${ }^{13}$

Insieme operano i criminali e la polizia, i profittatori di guerra con le presunte, a volte false, vittime, la malavita e gli operai, ciascuno facendo finta di non sapere niente delle attività degli altri. $O$ per il cinismo o per una specie di umorismo noir dell'autore, il paradosso si afferma come abituale, essendo percepito come frutto delle mutazioni moderne dello spazio urbano. Così, ad esempio, mentre il commissario indaga e cerca invano i colpevoli trascorrendo giorni e notti in giro per i quartieri della città, correndo il rischio di essere aggredito, ferito o licenziato, è all'oscuro del fatto che è proprio a casa sua, durante le cene da lui stesso organizzate, che si radunano quegli stessi colpevoli. Sono i protagonisti delle attività illegali che lui intende smascherare. Suo figlio, ad esempio, un animalista convinto, partecipa ad azioni segrete e incide graffiti scontrandosi con la polizia, mentre suo padre crede che lavori in un ristorante. La sua amica antiquaria, all'apparenza una tranquilla signora anziana, di notte si traveste da giovane barbona e va in giro a incidere graffiti. L'umorismo e il paradosso scaturiscono dal rovesciamento della realtà e della sua percezione e da alcuni momenti bizzarri, riconducibili soprattutto a una dettagliata rappresentazione dei personaggi e a una certa stravaganza nella descrizione del tempo e del luogo. L'ambiente triestino viene minuzionsamente analizzato da Heinichen che ne mette in evidenza i molti contrasti che perdurano nel tempo.

\section{Il quarto elemento: la società e gli esponenti della malavita}

In seguito alla scoperta di un deposito di armi, il commissario scopre alcuni segreti del passato che neanche immaginava potessero portarlo allo scontro con gli interessi dei Cavalieri di Malta e addirittura con il Vaticano, che, come tutti i poteri forti, lo bloccano ogni volta che si avvicina alla verità. La sua amica Graziella racconta dei criminali di guerra che furono fatti emigrare clandestinamente e che, per sfuggire alla giustizia, si nascondevano nei monasteri croati e italiani. Laurenti si rende conto che «la maggior parte delle cose in questa città sono intrecciate con il passato», ${ }^{14}$ con una coincidenza quasi

13 Ivi, p. 47.

14 Ivi, p. 74. 
assurda tra le persone e i luoghi. Graziella riferisce, inoltre, di spie e di servizi segreti nel Territorio libero sotto l'amministrazione alleata, della guerra fredda e dei riflessi della cortina di ferro, del trasferimento di cittadini benestanti e dei flussi migratori, e dei destini di intere famiglie che si spostavano per il timore di un futuro incerto. L'ipoteca del passato pesa molto sul presente, per cui la città non si libera facilmente dallo stigma del figlio indesiderato e vi fiorisce la criminalità organizzata che non conosce ideali né confini ed è magari anche protetta dai servizi segreti. L'attualità non può ignorare quanto accaduto nei tempi passati. Non a caso il procuratore insiste che il presente ha quasi sempre a che fare con il passato. Anche Laurenti, esaminando i vecchi casi di polizia conservati in due fascicoli, si chiede il perché dei ricatti connessi a vicende di sessant'anni prima. Qualora dovessero morire i testimoni, la verità potrebbe continuare a vivere solo nei libri e, prima o poi, avrebbe assunto aspetti diversi, al punto da essere deformata. Alla fine, si sarebbe persa per sempre:

Ormai la società focalizzava il suo interesse sulla cattura dei nuovi criminali di guerra, anche se si interveniva sempre quando era ormai troppo tardi. Allora si dava la caccia a un paio di sgherri, mentre dietro le quinte restava tutto come prima. L'occidente era troppo satollo, pigro e impotente. Alla gente mancavano sia la fantasia sia la volontà di cambiare qualcosa. Si guardava di sottecchi ai profitti futuri, e si spartivano gli affari e si restava in attesa. ${ }^{15}$

Nel finale, che si dirama in più direzioni, cresce la suspence. Tra l'altro Heinichen si sofferma sulla prospettiva della criminalità organizzata e, rovesciando il punto di vista, offre l'immagine del mondo visto dall'occhio di un criminale.

Veniamo a conoscere infatti il ragionamento del boss della zona, Viktor Dragič, la cui malvagia reputazione precede le sue azioni criminose. Crudele con i membri della sua banda, con emarginati, sordomuti, ladri, assassini, egli mostra notevole astuzia quando vuole imporsi su un concorrente. Con il solo obiettivo di arricchirsi e completamente senza scrupoli, Dragič ricatta, traffica con i clandestini, pianifica lo spostamento delle rotte cinesi da Belgrado a Tirana, colpendo o eliminando coloro che si mettono sulla sua strada, o che egli semplicemente sospetta che vogliano farlo. Una crudeltà incredibile si manifesta, ad esempio, nei confronti di una ragazza sordomuta, da parte delle 'teste rasate', descritte da Branka, una ragazza che appartiene allo stesso milieu criminale, ma che ne è anch'essa particolarmente impressionata:

15 Ivi, p. 320. 
Le avevano tagliato i capelli e poi rasato barbaramente la testa. L'avevano legata al letto con braccia e gambe aperte e poi passato una corda intorno alla gola annondandola stretta ai lacci delle gambe, tanto che non poteva muoversi di un millimero senza strangolarsi. Avevano appeso uno specchio sopra il letto. In un primo tempo Irina quasi non si riconobbe, poi si spaventò per le ferite che la lama del rasoio le aveva inferto sul viso e sulla pelle della testa. Le avevano comunicato che non le avrebbero dato né da mangiare né da bere finché non si rabboniva, e che non sarebbe mai riuscita a fuggire. ${ }^{16}$

Branka è descritta come una ragazza intelligente, una professionista dal fisico palestrato e d'aspetto gradevole. Chi la sottovalutava, pagava con la vita. Nel vedere la sordomuta Irina con la testa insanguinata e l'occhio tumefatto, Branka comincia a dubitare, e quindi assistiamo ad un suo mutamento, provocato dalla solidarietà femminile o da un'umanità ritrovata o dal semplice istinto di conservazione. In ogni caso Branka cambia aspetto e, attribuendole qualità più 'umane', l'autore la rende quasi simpatica e molto vicina al lettore. Le due donne sono all'opposto: Irina, estremamente debole, vittima della violenza o di ripetuti maltrattamenti; Branka, una ragazza clandestina forte, svelta e abile, capace di agire in modo rapido e duro nel momento giusto, la cui forza fisica e mentale è tremenda. Va ammirata, a questo riguardo, l'abilità dell'autore nel descrivere nei minimi dettagli l'ambiente clandestino, non solo quanto vi accade ma anche la psicologia di chi vi si muove.

Veit Heinichen, che, come egli stesso confessa, con il suo protagonista Proteo Laurenti ha una sola cosa in comune, la realtà dei luoghi descritti nei suoi romanzi, è un autore consapevole del contesto politico, storico e sociale in cui vive e con uno sguardo allargato all'orizzonte europeo, e ai problemi che vi coglie, soprattutto quelli relativi all'alta finanza, alle speculazioni economicofinanziarie, al traffico di esseri umani e di sostanze stupefacenti. Sono problemi, questi, che egli affronta, sulla scorta di una notevole informazione, attraverso la forma del noir.

Va quindi ammirata l'abilità dell'autore nel descrivere e mediare magistralmente tutte le sfumature dell'ambiente clandestino, a partire dalla descrizione dei conflitti alla psicologia dei personaggi e al modo di agire dei protagonisti. Il noir, in tal senso, si presenta - nella versione di Veit Heinichen il veicolo perfetto per esplorare l'attualità nelle sue molteplici sfaccettature, aggiungendo al classico schema triangolare lo spazio quale ulteriore elemento di rappresentazione.

16 Ivi, p. 342. 
